\title{
Primary renal lymphoma with a large mass: A case report and a review of the literature
}

\author{
Mazaher Ramezani ${ }^{1}$, Pourya Ghobadi ${ }^{2}$, Alireza Sadeghsalehi ${ }^{2}$, Khashayar Rahmani ${ }^{1}$, Masoud Sadeghi ${ }^{3}$ \\ (1) Kermanshah University of Medical Sciences, Molecular Pathology Research Center, Imam Reza Hospital, \\ Kermanshah, Islamic Republic of Iran
}

(2) Kermanshah University of Medical Sciences, Students Research Committee, Kermanshah, Islamic Republic of Iran

(3) Kermanshah University of Medical Sciences, Medical Biology Research Center, Kermanshah, Islamic Republic of Iran

Date submitted:

Dec 02, 2018

Date accepted:

Jul 10, 2019

Online publication date:

December 15, 2019

\section{Corresponding Author:}

Masoud Sadeghi

Kermanshah University of

Medical Sciences, Medical

Biology Research Center,

Kermanshah, Islamic Republic

of Iran.

sadeghi_mbrc@yahoo.com

Keywords: Primary renal

lymphoma, immunohistochemistry, renal tumor, case report.

\section{ABSTRACT}

Primary renal lymphoma (PRL) is uncommon and a controversial manner due to lack of the lymphatic tissue in the kidneys. Less than $1 \%$ of the kidney lesions are PRL. We reported a 61-year-old female with abdominal and left flank pain. She had a large renal mass measured $19 \mathrm{~cm}$. The imaging, histopathology, and immunohistochemistry diagnosed the large mass as primary renal lymphoma, diffuse large B-cell type.

\section{Introduction}

Primary Renal Lymphoma (PRL) is a very rare disease, as a non-Hodgkin's lymphoma (NHL), which primarily involves kidney with no metastasis from other sites of the body (1). Diffuse large B-cell lymphoma (DLBCL) is the main subtype with about $40 \%$ of cases (2). In adults, one kidney is usually involved in PRL, but bilateral involvement is observed in pediatrics (3). Finally, the disease will progress beyond the kidneys and few people with a disease live more than one year (4). It is suggested that PRL originates from the lymph nodes of the kidney sinuses or the lymphatic system of the kidney capsule (2). We reported a 61-year-old woman diagnosed with PRL.

\section{Presentation of Case}

A 61-year-old female was admitted to the general hospital in Harsin city, Kermanshah province, Iran, on 13th June 2017 with abdominal and left flank pain. There was only mild dysuria. The patient was diabetic. The ultrasound examination revealed hypo echoic left kidney mass measuring $3^{*} 6.1 \mathrm{~cm}$. The mild fatty liver change was noted. The uterus and adnexa were atrophy. There was no lymphadenopathy. Otherwise liver, kidneys, and spleen were normal. The multi slice spiral computed tomography (CT) scan of abdomen and pelvis with contrast on 31th July 2017 reported massive and heterogeneous mass measuring $12 \mathrm{~cm}$ in the left upper kidney with renal vessel involvement suggestive for renal space occupying lesion. Other intra abdomen organs were unremarkable. No significant para-aortic lymphadenopathy was noted. The lab data before surgery included Blood sugar: $247 \mathrm{mg} / \mathrm{dl}$, while WBC count, Ca, Albumin, Alkaline phosphatase, Na, K, Urea, Creatinine, Direct and Total bilirubin were within normal limits. The patient underwent radical nephrectomy on 5th September 2017. Very large adhesive mass in fascia gerota was present. The specimen was sent to the pathology department and consisted of three separate fragments. A deformed kidney labeled as left kidney was measured $14^{*} 9^{*} 7 \mathrm{~cm}$. The ureter was unrecognizable. In cut sections, several hemorrhagic areas were seen and the parenchyma was deformed. Mass was infiltrated in perirenal fat. The tumor diameter in the kidney was $12 \mathrm{~cm}$. Two other fragments totally measured $7 * 4 * 3 \mathrm{~cm}$ and had several hemorrhagic areas in cut sections was also submitted (Figure 1). The nephrectomy pathology diagnosis was "DLBCL of kidney extending to perirenal 
fat, high grade" (Figure 2). The Hilum was involved by tumor. Tumor greatest diameter was $19 \mathrm{~cm}(12+7 \mathrm{~cm})$.

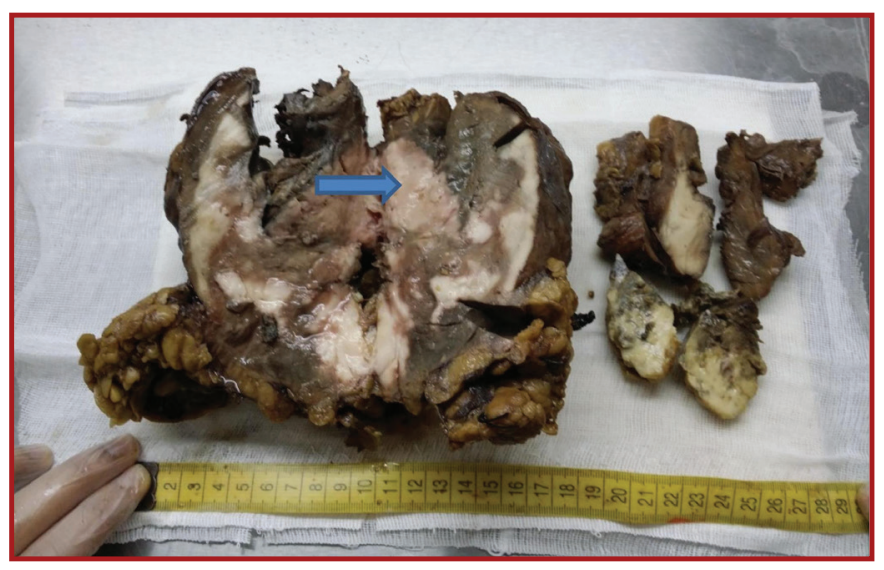

Figure 1. Gross pathology of the mass. Portion of tumoral component is shown by arrow.

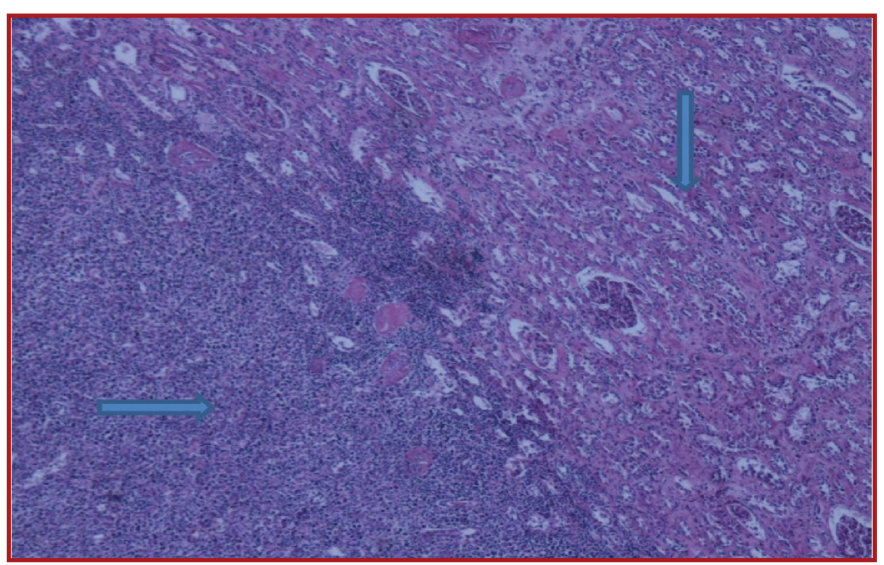

Figure 2. Hematoxylin-Eosin staining (x40 magnification). Massive infiltration of large lymphoid tumoral cells (Horizontal arrow) in the kidney (Vertical arrow)

Immunohistochemistry demonstrated Leukocyte common antigen (LCA), Cluster of differentiation (CD) 20 (Figure 3), and Vimentin positivity, whereas cytokeratin (CK), CD3, Inhibin-alpha, Melan-A, CK7, CD10, and Chromogranin negativity. As the final diagnosis, pathologist reported DLBCL with the renal involvement. The patient was in good condition on 29th October 2017 and referred for the complementary treatment.

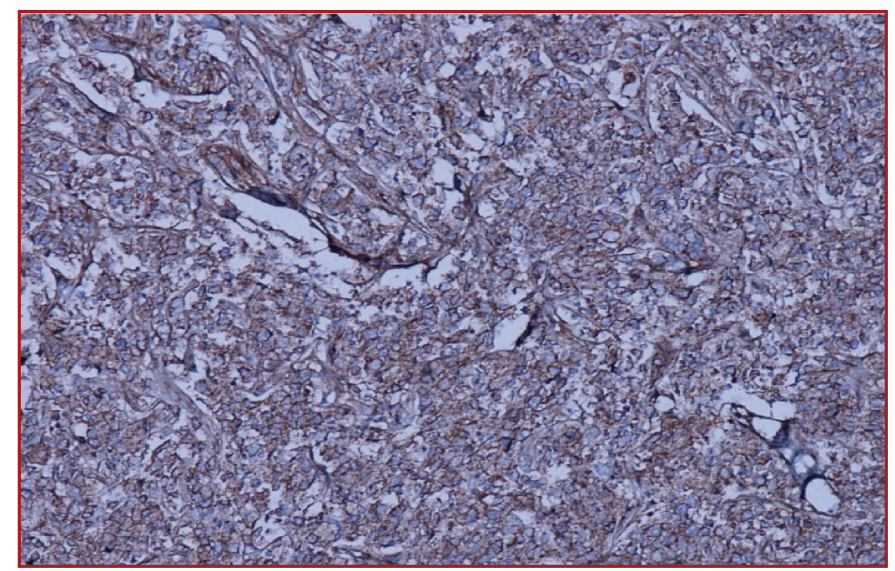

Figure 3. Immunohistochemistry of CD20 (x 100 magnification). Membrane brown staining of large lymphoid tumoral cells.

\section{Discussion}

In many countries, between $25 \%$ and $30 \%$ of $\mathrm{NHL}$ originates from extranodal areas (1). Kidneys can be initially involved, or a place for advanced extranodal involvement (5). During the diffuse stage of $\mathrm{NHL}$ renal involvement is not rare, with an incidence of $47 \%$ in autopsy (2). PRL is uncommon and a controversial manner because the kidneys do not have lymphatic tissue. It is suggested that the pathogenic mechanism involves lymphatic origin of the kidney capsule or subcapsular lymph tissue that invades the renal parenchyma or any chronic inflammatory process can be the cause of lymphoma (6). Less than $1 \%$ of the kidney lesions are PRL. In $10 \%$ to $20 \%$ of cases, there is a bilateral involvement (4).

PRL has no certain clinical presentations and it seems like kidney abscess, renal cell carcinoma (RCC) or metastasis to the kidneys (3) and also clinical manifestations are similar to other kidney cancers. The most common clinical symptoms are abdominal or flank pain (7). This case was presented with abdominal and left flank pain. Other symptoms and signs were hematuria, abdominal mass, and systemic symptoms including fatigue, weight loss, and fever (1). PRL can be associated with nephrotic syndrome or proteinuria, and especially in cases where two kidneys are involved, it may immediately develop to renal failure (7). In addition to abdominal and left flank pain, our patient only had a mild dysuria.

After recognition of renal tumor, the spread of extra-renal lymphoma is seen in nearly all cases that have PRL (8). DLBCL forms a large part of subdivision of the $\mathrm{NHL}$, and in the West, $30 \%$ of all cases of lymphoma are included (1). Many forms of involvement have been introduced including: One or more separate masses, spreading penetration leading to the significant large organ and intravascular involvement pattern (4).

The CT scan, ultrasound, IV urography, Magnetic Resonance Imaging (MRI), and Nuclear imaging are multiple imaging choices for checking kidney involvement (5). In ultrasound, $\mathrm{PRL}$ is seen as a hypoechoic tumor (3) like the present case. Lymphoma is seen in the CT scan in different patterns as single tumor (Less than $6 \%$ of cases), multiple kidney tumors $(60 \%)$ ,retroperitoneal lymphoma that invades kidney (25-30\%) and around or infiltrating kidney (25\% - 30\%) (5). In our patient, the CT scan demonstrated a massive and heterogeneous mass in left upper kidney with renal vessel involvement suggestive for renal space occupying lesion. PRL in MRI shows low intensity on T1-weighted views and isointensity to low intensity on T2-weighted views (3).

Today for the treatment of PRL, systemic chemotherapy is used as a first choice (7). The CHOP (cyclophosphamide, doxorubicin, vincristine, and prednisone) regimen is the selective treatment choice for $\mathrm{PRL}$ patients, which, if possible, associated with rituximab (R-CHOP). A better prognosis is seen with the recent regimen (4). Butani et al. (9) reported a 12-year -old boy with diagnosis of PRL, who treated with cyclophosphamide, vincristine, prednisone, and intrathecal methotrexate. After non-response, additional doses of vincristine, prednisone, methotrexate, doxorubicin, etoposide, and intrathecal methotrexate was started.They achieved a good response with the later regimen. Napoli et al. (10) reported a 53-year-old female with bilateral PRL, B-cell type, that 6 cycles of R-CHOP-21 protocol was used and achieved a good response in a follow-up of 1.5 years. 


\section{Conclusions}

$\mathrm{PRL}$ is rare, but may be seen as large renal mass. The pathologist and surgeon must be aware of this entity and consider it in the differential diagnosis of renal masses.

\section{Acknowledgments}

Author contributions MR: Conception, design, data collection, manuscript writing, supervision; MS: Literature review, manuscript writing; PG \& AS: Analysis and interpretation of findings, manuscript writing; KR: critical review. In addition, the authors would like to thank the Clinical Research Development Center of Imam Reza Hospital for Consulting Services.

\section{Conflict of Interest}

The authors declared they do not have anything to disclose regarding conflict of interest with respect to this manuscript.

\section{References}

1. Ladha A, Haider G. Primary renal lymphoma. J Coll Physicians Surg Pak. 2008;18(9):584-585.

2. Hagihara M, Hua JZ, Iwaki $Y$, Inoue M, Sato T. Primary renal lymphoma: a case report and literature review. Intern Med. 2015;54(20):2655-2659.

3. Chen X, Hu D, Fang L, et al. Primary renal lymphoma: A case report and literature review. Oncol Lett. 2016;12(5):4001-4008.

4. Shetty S, Singh AC, Babu V. Primary Renal Lymphoma - A Case Report and Review of Literature. J Clin Diagn Res. 2016;10(9):XD05-XD07.

5. Pinggera G-M, Peschel R, Buttazzoni A, Mitterberger M, Friedrich A, Pallwein L. A possible case of primary renal lymphoma: a case report. Cases J. 2009;2:6233.

6. Geetha N, Shahid A, Rajan V, Jacob PM. Primary renal lymphoma-a case report. Ecancermedicalscience. 2014;8:466.

7. Vázquez-Alonso F, Puche-Sanz I, Sánchez-Ramos C, Flores-Martín J, Vicente-Prados J, Cózar-Olmo JM. Primary renal lymphoma: long-term results of two patients treated with a chemotherapy + rituximab protocol. Case Rep Oncol Med. 2012;2012:726424.

8. Wang Y, Guo S. Primary renal diffuse large B-cell lymphoma with central nervous system involvement: a rare case report and literature review. Int J Clin Expl Pathol. 2015;8(6):7045-7049.

9. Butani L, Ducore J. Primary Renal Lymphoma Presenting as End-Stage Renal Disease. Case Rep Med. 2017;2017:9210648.

10. Napoli M, Montinaro AM, D'Ambrosio E, et al. [The bilateral renal lymphoma: an incurable disease? Case report]. G Ital Nefrol. 2014;31(1). pii: gin/31.1.13. Italian. 